BNL - 71229-2003-IR

\title{
Soft X-ray FEL by Cascading Stages of High Gain Harmonic Generation
}

\author{
Li Hua Yu \\ National Synchrotron Light Source \\ Brookhaven National Laboratory \\ Upton, New York 11973
}

April 2003

\section{National Synchrotron Light Source}

\author{
Brookhaven National Laboratory \\ Operated by \\ Brookhaven Science Associates \\ Upton, NY 11973 \\ Under Contract with the United States Department of Energy \\ Contract Number DE-AC02-98CH10886
}




\section{DISCLAIMER}

This report was prepared as an account of work sponsored by an agency of the United States Government. Neither the United States Government nor any agency thereof, nor any of their employees, nor any of their contractors, subcontractors or their employees, makes any warranty, express or implied, or assumes any legal liability or responsibility for the accuracy, completeness, or any third party's use or the results of such use of any information, apparatus, product, or process disclosed, or represents that its use would not infringe privately owned rights. Reference herein to any specific commercial product, process, or service by trade name, trademark, manufacturer, or otherwise, does not necessary constitute or imply its endorsement, recommendation, or favoring by the United States Government or any agency thereof or its contractors or subcontractors. The views and opinions of authors expresses herein do not necessarily state to reflect those of the United States Government or any agency thereof. 


\title{
Soft X-ray FEL by Cascading Stages of High Gain Harmonic Generation
}

\author{
Li Hua Yu \\ NSLS, BNL
}

\section{Introduction}

Short wavelength Free-Electron Lasers are perceived as the next generation of synchrotron light sources. In the past decade, significant advances have been made in the theory and technology of high brightness electron beams and single pass FELs. These developments facilitate the construction of practical VUV FELs and make x-ray FELs possible. Self-Amplified Spontaneous Emission (SASE) [1-16] and High Gain Harmonic Generation (HGHG)[17-19] are the two leading candidates for x-ray FELs. The first lasing of HGHG proof-of-principle experiment[20,21] succeeded in August, 1999 in Brookhaven National Laboratory. The experimental results agree with the theory prediction. Compared with SASE FEL, the following advantages of HGHG FEL were confirmed; 1. Better longitudinal coherence, and hence, much narrower bandwidth than SASE. 2. More stable central wavelength, 3. More stable output energy.

In this introduction, we will first briefly describe the principle of HGHG in Section A. Then in Section B, we give a general description about how to produce soft $\mathrm{x}$-ray by cascading HGHG scheme. In section 2, we give a detailed description of the system design. Then, in section 3 . we give a description of an analytical estimate for the HGHG process, and the calculation of the parameters of different parts of the system. The estimate is found to agree with simulation within about a factor 2 for most cases we studied. The stability issue, the sensitivity to parameter variation, the harmonic contents of the final output, and the noise degradation issue of such HGHG scheme are discussed in Section 4. The results are presented in Section 4. Finally, in Section 5, we will give some discussion of the challenges in development of the system. The conclusion is given in Section 6.

\section{A. The HGHG principle}

The HGHG scheme evolved from earlier and related ideas[22-33]. In the HGHG scheme, there are three components, i.e., one undulator used as the modulator, one dispersion section, and a second undulator used as the radiator. As in Fig. 1, a seed laser, together with an electron-beam, is introduced into the modulator. So, in the modulator, the seed laser interacts with the e-beam, and energy modulation is formed in the e-beam. Then the energy-modulated e-beam passes through the dispersion section (a three-dipole chicane), where the energy modulation in the ebeam is converted into spatial modulation. 
Abundant harmonics exist in such spatially modulated e-beam. Then such spatially modulated ebeam enters the radiator. The radiator is designed to be resonant to one of the harmonics of the seed laser. So, once the spatially modulated e-beam enters the radiator, rapid coherent emission at this resonant harmonic is produced, and then, this harmonic is further amplified exponentially until saturation. This is exactly the main set-up for the first HGHG experiment [20-21]. In this experiment, the input $\mathrm{CO}_{2}$ seed laser power was $0.7 \mathrm{MW}$ at $10.6 \mu \mathrm{m}$ the output HGHG FEL power was about $35 \mathrm{MW}$ at $5.3 \mu \mathrm{m}$. Compared with the seed laser, Harmonic Generation was achieved, i.e. the second harmonic was radiated. Also, High Gain was confirmed, the SASE pulse energy was measured to be 10 times larger than the spontaneous radiation and the HGHG pulse energy was $10^{7}$ times as large as the SASE signal, which, in the case of the HGHG experiment, provides a background noise. This experiment proved the principle of the HGHG FEL, and also demonstrated the advantages of HGHG FEL.

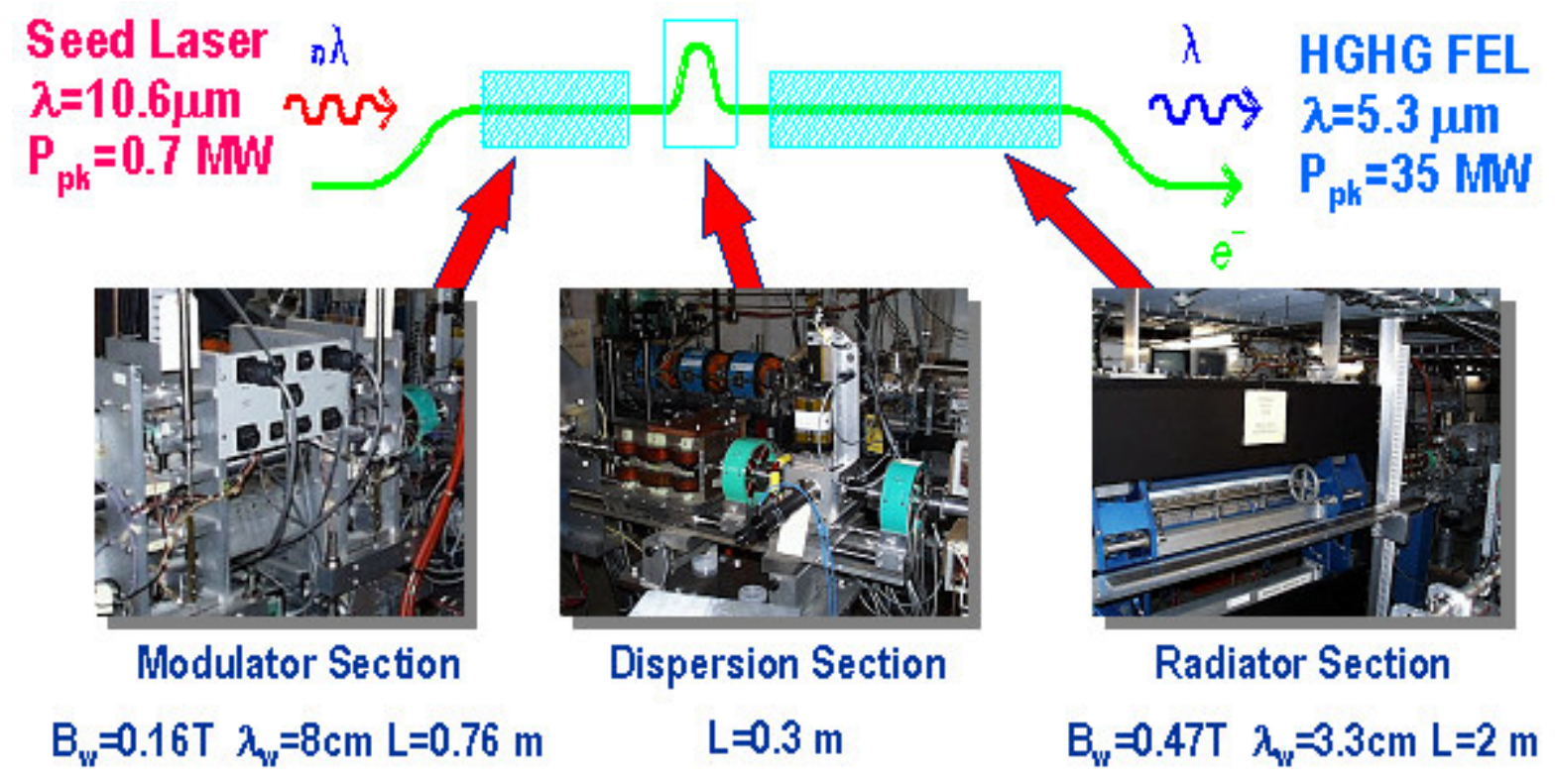

Electron Beam Input Parameters: $E=\mathbf{4 0} \mathrm{MeV}$

$\varepsilon_{\mathrm{n}}=4 \pi \mathrm{mm}-\mathrm{mrad} \mathrm{d} \gamma / \gamma=0.043 \% \mathrm{I}=110 \mathrm{~A} \tau_{\mathrm{e}}=4 \mathrm{ps}$

Fig. 1 Proof of Principle Experiment Setup at BNL

In order to distinguish the relative local energy spread before the e-beam enters the undulator from the relative local energy spread growth due to the quantum diffusion effect [34] when the ebeam traverses the undulator, in our description, initial relative local energy spread refers to the value before the e-beam enters the undulator. So in Fig. $1, \sigma_{\gamma} / \gamma=0.043 \%$ is the initial relative local energy spread. 


\section{B. The HGHG scheme to produce soft x-ray}

We now describe the approach to generate soft $x$-ray by cascading HGHG stages [35,36]. Cascading two stages of HGHG for soft x-ray FEL to $6 \mathrm{~nm}$ has been proposed before [37,38]. To reach $2.1 \mathrm{~nm}$, we need more than two stages and there are new issues to be addressed here.

There exist commercially available lasers with wavelength about thousands Angstrom. On the other hand, we hope to produce x-ray with a wavelength around several Angstroms. To achieve $2.1 \mathrm{~nm}$ by one step of HGHG would require very high harmonic of the order of several hundreds. Previous analysis [37]showed that to generate high harmonic, one needs very high input seed laser power. Beyond 60'th harmonic, this becomes difficult. Also, as we will discuss in the section 4 of this paper, when harmonic is too high, stability of the output is not good. So, we need some modification

1. We need multiple stages. During each stage the n'th harmonic of the seed laser will be produced at the end of the radiator, and then this harmonic will be used as the seed for the next stage. In reality, $\mathrm{n}$ should not be too large. In our design we use $\mathrm{n}=5$ to achieve stable performance. So, if we start from available commercial laser of hundreds $\mathrm{nm}$ as the seed, we would need three stages to reach soft $\mathrm{x}$-ray with a wavelength of several $\mathrm{nm}$.

2. Conceptually, the device is composed of two parts. A converter, and an amplifier. The converter consisting of several stages, converts the seed laser to the designed wavelength step by step. Then at the end, an amplifier exponentially amplifies the radiation obtained from the last stage to saturation.

3.Except for the second stage and the last amplifier, each stage only converts the light to its n'th harmonics, and there is almost no exponential growth.

4.Since we need cascade several stages of HGHG, we need some extra components. Each stage will be the same as shown in Fig. 1, i.e., each stage will consist of one modulator, a dispersion section, and one radiator. The physics process in each stage will be the same as in the recent experiment [20,21]. During the process, the output radiation has disturbed a part of e-beam, which coincides with it. So in order to achieve best efficiency to carry out the next stage of HGHG, we need use a fresh e-beam. For this purpose, after one stage of HGHG, we shift the laser (i.e., the output radiation from the previous HGHG stage) to the front part of the same ebeam, so that the laser will interact with a "fresh" part of the same e-beam. This is the "fresh bunch technique" [37]. This is schematically plotted in Fig. 2. We use a chicane (a "shifter") to shift the laser to the "fresh" part of the same e-beam.

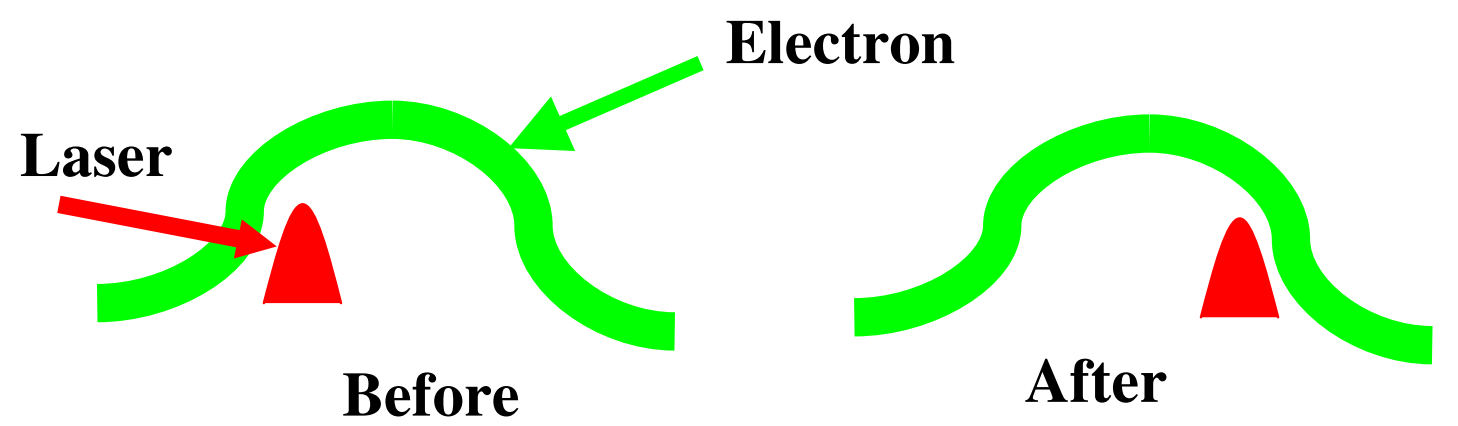

Figure 2. Fresh bunch technique 


\section{Description of the system}

Let us now present the details of the whole device. As shown in Fig. 3, we consider an available laser with a wavelength of $266 \mathrm{~nm}$, and a peak power of $\mathrm{P}_{\mathrm{in}}=500 \mathrm{MW}$, and pulse length of $20 \mathrm{fs}$. The corresponding start-up shot-noise power [10] is only about $\mathrm{P}_{\text {noise }}=30 \mathrm{~W}$. So, the input seed laser power dominates the shot-noise power. This is true for all seed lasers into the three stages and the last amplifier. This dominance is necessary, because even though there is only negligible noise power in the initial stage, the "signal-to-noise" ratio of the final radiation at $2.1 \mathrm{~nm}$ might be degraded [41]. Calculation (as will be explained in section 4) shows that a 500MW seed laser could ensure that the signal-to-noise ratio at the final $2.1 \mathrm{~nm}$ radiation to be around 1000 . After 3 stages, we have $2.1 \mathrm{~nm}$ radiation, and then this $2.1 \mathrm{~nm}$ radiation is amplified to the saturation region with a peak power around $1.7 \mathrm{GW}$ by traversing the last undulator, the amplifier.

The parameters for the electron beam, the undulators, and the dispersion section are given in the Figure 3 and the Table 1. Let us first explain the meaning of each parameter in Fig. 3. The number on the first row stands for the output power of each stage. The output power of one stage is the input power of the next stage, though diffraction effect should be taken into consideration as we will discuss shortly. The second row stands for the corresponding wavelength of the radiation. The e-beam parameters are printed just below the schematic device. The system has a betatron function of $6.8 \mathrm{~m}$.

The e-beam has a peak current of $750 \mathrm{Amp}$, an energy of $2.6 \mathrm{GeV}$, normalized emittance $\epsilon_{\mathrm{n}}=1$ mm-mrad, and initial relative local energy spread $\sigma_{\gamma} / \gamma=2 \times 10^{-4}$. Due to spontaneous radiation, this is increased. We further upgrade [47] our code to simulate the growing of the relative local energy spread along the undulator, and we found that the results obtained using the same relative local energy spread $\left(\sigma_{\gamma} / \gamma=2 \times 10^{-4}\right)$ agree well with the results given by the upgraded code, so the effect is negligible.

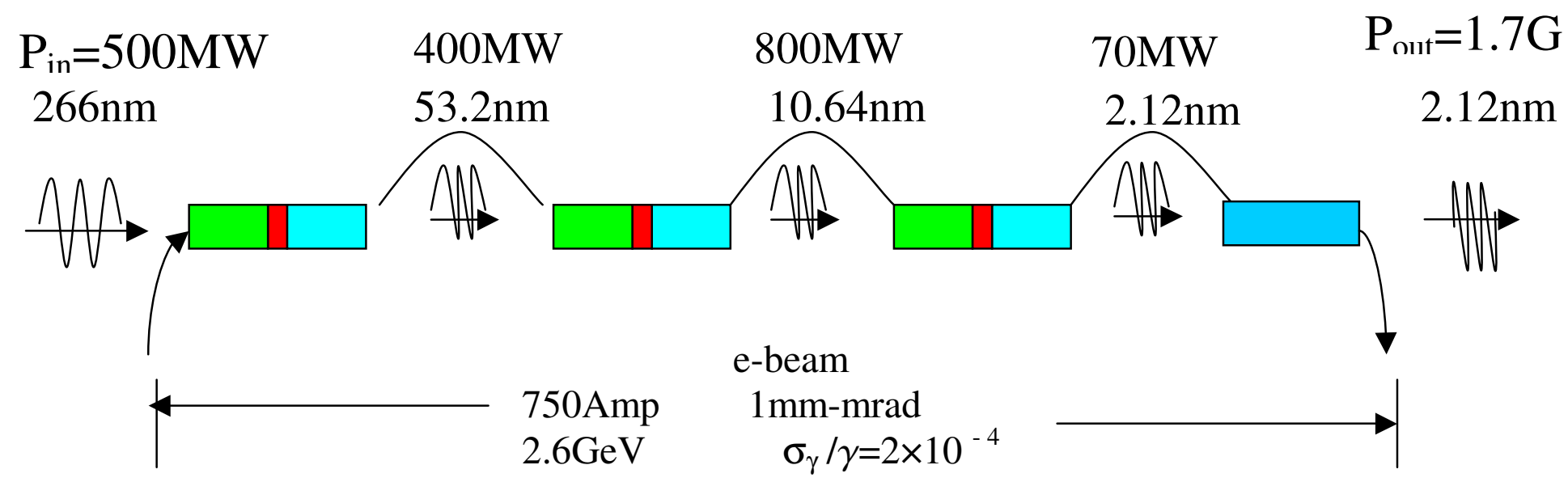

Figure 3. 


\begin{tabular}{|c|c|c|c|c|c|c|c|}
\hline & & & $2^{\text {nd }}$ & & $3^{\text {rd }}$ & & Amplifier \\
\hline$\lambda(\mathbf{n} \mathbf{m})$ & 266 & 53.2 & 53.2 & 10.64 & 10.64 & 2.128 & 2.128 \\
\hline$\lambda_{w}(\mathbf{c m})$ & 11 & 6.4 & 6.4 & 4.16 & 4.16 & 2.7 & 2.7 \\
\hline $\mathbf{d} \psi / \mathbf{d} \gamma$ & & & & & 0 & & \\
\hline $\mathbf{L}_{\mathrm{w}}(\mathbf{m})$ & 2 & 6 & 2 & 8 & 2 & 4 & 12 \\
\hline $\mathbf{L}_{G}(\mathbf{m})$ & 1.6 & 1.3 & 1.3 & 1.4 & 1.4 & 1.75 & 1.75 \\
\hline
\end{tabular}

$\mathrm{L}_{\text {total }}=36 \mathrm{~m}$ to reach $1.7 \mathrm{G} \mathrm{W}$

Table 1

For the table, the fist row gives the radiation wavelength. The second row is the undulator period. The third row is the dispersion strength. The fourth row is the length of the undulators (modulators, radiators, and the amplifier). For an example, the last amplifier has a length of 12 $\mathrm{m}$. The fifth row stands for the power e-folding length in each undulator without energy modulation. The table has 4 boxes, the first three stand for the three harmonic generation stages, while the last one stands for the amplifier. In each of these boxes, which stands for each stage, the left column gives the parameters for the modulator, while the right column gives those for the radiator. The numbers in the middle, stand for the dispersion strength $\mathrm{d} \psi / \mathrm{d} \gamma(\psi$ is the phase in the radiator).

For an example, the second box stands for the second stage. The left column in this second box stands for the modulator of the second stage. The table shows that in the modulator the resonant radiation has a wavelength of $53.2 \mathrm{~nm}$, the modulator has a period of $6.4 \mathrm{~cm}$, the length of the modulator is $2 \mathrm{~m}$, and the corresponding power e-folding length without energy modulation is $1.3 \mathrm{~m}$. The right column shows that the radiation in the radiator has a wavelength of $10.64 \mathrm{~nm}$, the radiator has a period of $4.16 \mathrm{~cm}$, the length of the radiator is $8 \mathrm{~m} \mathrm{long}$, while the corresponding power e-folding length is $1.4 \mathrm{~m}$. The numbers in the middle, i.e. 1.0, stands for the dispersion strength $\mathrm{d} \psi / \mathrm{d} \gamma$, and energy spread $2 \times 10^{-4}$. Similarly for the other boxes, except for the fourth box. The fourth box stands for the amplifier, so there is no dispersion strength $\mathrm{d} \psi / \mathrm{d} \gamma$. The effect of the global energy spread (or correlated energy spread, in the terminology of certain other workers in this field) is addressed in the following discussion of the sensitivity to the parameter variation, for its effect is essentially an issue of detuning. 
Now, let us explore the physics process in such device. As shown in Fig. 3, the $266 \mathrm{~nm}$ laser, with a peak power of $500 \mathrm{MW}$, together with the $2.6 \mathrm{GeV}$ e-beam, are introduced into the modulator of the first stage. So an energy modulation is formed in the e-beam. Then by passing through the following dispersion section, the energy modulation is converted into a spatial modulation. Such a spatially-modulated e-beam will then be introduced into the following radiator. The radiator is resonant to the fifth harmonic of the seed laser, so we will have $53 \mathrm{~nm}$ coherent emission to reach $400 \mathrm{MW}$. The length of the radiator is only four gain lengths so there is almost no exponential growth. In order to go to next stage, we need a shifter, in which the ebeam is magnetically delayed by a small chicane. Therefore effectively, the $53 \mathrm{~nm}$ radiation is shifted by 40 fs to the front part of the same e-beam, where the e-beam is still "fresh". To have the electron path longer than the straight line by $40 \mathrm{fs}$, the chicane is 0.35 meter long, with maximum field of 1.5 Tesla.

For the $2.6 \mathrm{GeV}$ e-beam and the parameters we choose for the undulators, we calculated the energy spread increase induced by spontaneous radiation in the undulator, or the quantum diffusion effect [34], and found the effect is negligible, as we mentioned before. So we continue to use the same energy spread of $\sigma_{\gamma} / \gamma=2 \times 10^{-4}$.

Now, the $53 \mathrm{~nm}$ radiation serves as the seed laser in the second stage, where the $53 \mathrm{~nm}$ radiation input generates a $10.6 \mathrm{~nm}$ output with $800 \mathrm{MW}$. Next, after passing through another shifter to interact with the fresh part of the electron bunch, the $10.6 \mathrm{~nm}$ radiation is the seed laser for the next stage to be converted to $2.1 \mathrm{~nm}$. Here, we would like to emphasize that, for the first and third stage, the radiators work at the coherent emission region, i.e. after the coherent emission is finished, the radiation is introduced to the next stage almost without exponential growth. This is the key point to make the total length of the device short. Finally, the $2.1 \mathrm{~nm}$ is again shifted to a fresh part of the electron bunch to be amplified exponentially to deep saturation at $1.7 \mathrm{GW}$ in the last undulator, i.e. the amplifier, of the device.

We emphasize, in the radiator for the first and third stages, there is almost no exponential growth of the harmonic, but rather, after the coherent emission is finished, the harmonic is introduced to the next stage directly. For an example, the length of the 3rd radiator is only $4 \mathrm{~m}$ long, while the corresponding power e-folding length without energy modulation is about $1.4 \mathrm{~m}$. With energy modulation in the harmonic generation process, the gain length is futher increased, so no exponential growth is expected.

When the radiation traverses the shifter, there is diffraction loss in the radiation, and will be discussed in the following section. The coherent synchrotron radiation effect (CSR) in the shifter and dispersion magnets has been estimated to be negligible. The path length changes in these chicanes are much smaller than the electron bunch compressor. 


\section{System Design Parameters}

\section{A. General criteria}

The following is a description of the physics process in each stage. As is shown in Fig. 3 (by comparing wiggler length with the power e-folding length), the modulators of all the stages and the radiators in stages 1, 3 are not working in exponential region. For these sections, it is justified to analyze the process ignoring the FEL interaction, i.e., calculate the radiation in terms of a known electron beam distribution, and ignore the reaction of the radiation to the electrons). Some further details of the derivation will be given in the following section. Here a brief qualitative description is given first.

As described in the HGHG principle section, in each HGHG stage, when the electron bunch enters the radiator, the harmonic contents at the seed laser wavelength and also its high harmonics are abundant. The radiator is designed to be resonant to a special harmonic, therefore coherent emission at this special harmonic is obtained. For the calculation of the n'th harmonic of the seed laser, the important quantity is the n'th harmonic coefficient of the spatial modulation, which is just two times the bunching factor [18]

$$
\boldsymbol{b}_{n} \equiv\left\langle\boldsymbol{e}^{i \psi}\right\rangle=\boldsymbol{e}^{-\frac{1}{2}\left(\frac{\partial \psi}{\partial \gamma} \sigma_{\gamma}\right)^{2}} \boldsymbol{J}_{n}\left(\frac{\partial \psi}{\partial \gamma} \Delta \gamma\right)
$$

where, $\psi=\boldsymbol{n} \theta$ is the ponderomotive phase in the radiator, with $\theta=\left(\boldsymbol{k}_{\boldsymbol{s}}+\boldsymbol{k}_{\boldsymbol{w}}\right) \boldsymbol{z}-\omega_{\boldsymbol{s}} \boldsymbol{t}$ the ponderomotive phase of the e-beam in the modulator. $\mathrm{k}_{\mathrm{s}}$ and $\omega_{\mathrm{s}}$ are the seed laser wave number and frequency, respectively. $\boldsymbol{k}_{w}=\mathbf{2} \pi / \lambda_{w}$, with $\lambda_{w}$ the period of the modulator. $\Delta \gamma$ is the energy modulation produced in the modulator, and $\sigma_{\gamma}$ is the rms local energy spread.

In the bunching factor, the exponential part is monotonous, therefore, to optimize the system, the parameters are adjusted so that $\mathrm{J}_{\mathrm{n}}(\mathrm{x})$ is near its maximum. Empirically, $\mathrm{J}_{\mathrm{n}}(\mathrm{x})$ peaks around $\mathrm{x} \sim 1.2 \mathrm{n}$ for $\mathrm{n} \geq 1$, hence the first criterion in design,

$$
\Delta \gamma\left(\frac{d \psi}{d \gamma}\right) \approx 1.2 n
$$

Now, the exponential part in the bunching factor reads,

$$
\exp \left[-\frac{1}{2} \sigma_{\gamma}^{2}\left(\frac{1.2 n}{\Delta \gamma}\right)^{2}\right]
$$

Obviously, the energy modulation should dominate the energy spread in order to obtain large bunching factor. So, it seems that the larger the modulation, the better the result. However, the larger the modulation, the larger the effective energy spread in the electron beam, this may reduce the radiation power. So, the exponent of Eq.(3A3) should be on the order of one, thus the second criterion is 


$$
\Delta \gamma \geq \boldsymbol{n} \sigma_{\gamma}
$$

Eq.s (3A2) and (3A4) are essentially the starting points of all our considerations, though some details need be considered in the real simulation. In the one stage case, as in the experiment of the references [20,21], Eq. (3A4) may not be satisfied, because we need to impose the limitation $\sigma_{\gamma} / \gamma<\rho$ (the Pierce parameter [2]) in order to ensure the exponential growth in the radiator. In the Harmonic Generation stages, i.e., in the converter, where exponential growth is not needed, $\Delta \gamma$ could be bigger than $\mathrm{n} \sigma_{\gamma}$, then the limitation is to avoid over-bunching in the radiators. We will explore more in the following sections.

\section{Analytical Estimate of HGHG}

The HGHG process can be separated into four distinct steps: the modulator, the dispersion section, the coherent harmonic generation in the first 2 gains length of the radiator, and the exponential growth section. The first three steps do not have much FEL interaction, i.e., the radiated power in these sections is so low that its reaction on the electron micro bunching is negligible. The exponential growth section has been studied extensively. These make it possible to estimate the process for each step separately. The estimate is good to within a factor of 2 when compare with simulation for most cases studied, giving generally correct dependence of output radiation and system parameters.

\section{B1. The modulator}

The modulator usually is designed less than 2 gain lengths, so the EM field in the modulator can be approximated to be constant throughout the undulator. Thus the maximum energy modulation at the end of the modulator can be written down using the FEL energy equation:

$$
\Delta \gamma=\frac{k_{s 1} a_{w 1} F_{B 1}}{\gamma} a_{s 1} z_{1} .
$$

Here $\gamma$ is the electron beam energy, $\boldsymbol{k}_{s \mathbf{1}}$ and $\boldsymbol{a}_{\boldsymbol{s 1}}$ are the wave-number $\mathbf{2} \pi / \lambda_{\boldsymbol{s 1}}$ and the dimensionless (rms) vector potential $\boldsymbol{e} \boldsymbol{A}_{s \mathbf{1}} / \boldsymbol{m} \boldsymbol{c}$ of the input laser field respectively, while $\boldsymbol{a}_{w 1}$, $\boldsymbol{F}_{\boldsymbol{B} 1}$, and $z_{1}$ are the dimensionless (rms) vector potential, the Bessel factor and the length of the modulator respectively $\left(\boldsymbol{F}_{B 1} \equiv \boldsymbol{J}_{0}(\eta)-\boldsymbol{J}_{1}(\eta)\right.$, with $\left.\eta \equiv \boldsymbol{a}_{w 1}^{2} / 2 /\left(1+\boldsymbol{a}_{w 1}^{2}\right)\right)$. Notice that since EM field is approximated as constant along the undulator, the energy change increases linearly along the undulator, so the integration with $\mathrm{z}$ is simply multiply by $\boldsymbol{z}_{\mathbf{1}}$, and the maximum energy change is at a phase $\theta_{1}=\pi / 2$, so that $\sin \left(\theta_{1}\right)=\mathbf{1}\left(\theta_{1}\right.$ is the phase in the modulator, as described in section A). Assuming the input laser is a gaussian beam, usually the modulator length is chosen to be comparable to the Rayleigh range $\boldsymbol{Z}_{\boldsymbol{R}}$ of the input laser, so the field variation along the undulator is order of less than $50 \%$, as a rough estimate we can take the value of $\boldsymbol{a}_{\boldsymbol{s 1}}$ to be the one at the entrance of the modulator and its transverse distribution is taken to be gaussian. We have $\boldsymbol{a}_{s 1}=\boldsymbol{a}_{s 1 m} \boldsymbol{e}^{-\frac{r^{2}}{w^{2}}}$ and if beam waist is at the centre of the modulator, at the entrance 
$w=w_{0} / \sqrt{1+\left(z_{1} / 2 / Z_{R}\right)^{2}}$ with $w_{0}=\sqrt{\lambda_{s 1} Z_{R} / \pi}$. As result, $\Delta \gamma$ has the same transverse dependence on radius $r$. However, except for the first stage, the input laser beam waist is set before the modulator because the input laser is just the output radiation of the previous HGHG stage. The calculation of the beam waist position is mentioned in section B7.

\section{B2. The Phase Advance and Bunching Parameters in the Radiators}

Next we describe the phase advance. In the radiator, the total phase advance for the electrons with the maximum energy modulation is

$$
\Delta \psi=\frac{\partial \psi}{\partial \gamma} \Delta \gamma
$$

where $\frac{\partial \psi}{\partial \gamma}$ is the total dispersion consists of the contribution from the modulator, the dispersion section, and the radiator:

$$
\frac{\partial \psi}{\partial \gamma}=\boldsymbol{n} \frac{\boldsymbol{k}_{w 1}}{\gamma} z_{1}+\left(\frac{\partial \psi}{\partial \gamma}\right)_{\text {dispersion }}+\frac{\boldsymbol{2 k}_{w 2}}{\gamma} z_{2} .
$$

The first and third terms are the dispersions in the modulator and the radiator respectively, with $\boldsymbol{k}_{w 1}, \boldsymbol{k}_{w 2}$ the respective undulator wavenumbers. The harmonic number $\mathrm{n}$ is present in the first term because the phase in the radiator is $n$ times the phase in the modulator. Also, in the first term, there is a factor $1 / 2$ introduced to cancel the factor 2 in the FEL phase equation as shown in the third term, because the energy modulation approximately increases linearly with the modulator distance $z_{1}$ thus the phase advance, obtained by integration over $z_{1}$, contributes a factor $1 / 2$.

The phase advance produces a micro-bunching with bunching parameter [18]

$$
\boldsymbol{b}_{n} \equiv\left\langle\boldsymbol{e}^{i \psi}\right\rangle=\boldsymbol{e}^{-\frac{\mathbf{1}}{\mathbf{2}\left(\frac{\partial \psi}{\partial \gamma} \sigma_{\gamma}\right)^{2}}} \boldsymbol{J}_{n}\left(\frac{\partial \psi}{\partial \gamma} \Delta \gamma\right)
$$

where $\sigma_{\gamma}$ is the rms energy spread, $\boldsymbol{J}_{\boldsymbol{n}}$ the n'th order Bessel function. Notice that $\boldsymbol{b}_{\boldsymbol{n}}$ is a function of radial position $\mathrm{r}$ (through $\Delta \gamma$ 's $\mathrm{r}$ dependence) and radiator distance $z_{2}$ because the radiator dispersion (the third term of Eq.3B3).

\section{B3. Coherent Power in the Radiators}

To calculate the radiation power in the radiator, we use a simplified model. For a flat top electron beam without energy spread and angular spread, but with a known initial bunching 
parameter $\boldsymbol{b}_{n}(\boldsymbol{r})$, the power is calculated as (derived based on a formula given by the reference [10]):

$$
\boldsymbol{P}=\boldsymbol{C} \quad \boldsymbol{P}_{c o h} \boldsymbol{e}^{\frac{z}{L_{G}}}
$$

where $\mathrm{z}$ is the distance in the radiator, $\boldsymbol{L}_{\boldsymbol{G}}$ the gain length, and the coupling coefficient $C \approx \frac{\mathbf{3 . 7 1}}{\mathbf{1 2}} \approx \frac{\mathbf{1}}{\mathbf{3}}$ in the large beam size limit (i.e., when the Rayleigh range which corresponds to the beam size is larger than the gain length), while $\boldsymbol{P}_{\boldsymbol{c o h}}$ is the coherently radiated power in first two gain lengths of the radiator:

$$
P_{c o h}=\frac{\left(I_{0}\right)^{2} Z_{0}}{8}\left(\frac{K F_{B 2}}{\gamma}\right)^{2} \frac{1}{4 \pi \sigma_{x}^{2}}\left|I_{b}\right|^{2}
$$

with $\boldsymbol{I}_{\mathbf{0}}$ the electron beam current, $\boldsymbol{Z}_{\mathbf{0}}=377 \Omega$ the vacuum impedance, $\boldsymbol{K}=\sqrt{\mathbf{2}} \boldsymbol{a}_{\boldsymbol{w} \mathbf{2}}$ the undulator parameter (in terms of maximum field) for the radiator, $\boldsymbol{F}_{\boldsymbol{B} \boldsymbol{2}}$ the Bessel factor, $\sigma_{\boldsymbol{x}}$ the rms electron beam size, and $\boldsymbol{I}_{\boldsymbol{b}}$ is the bunching integral over two gain lengths:

$$
I_{b}=\int_{0}^{2 L_{G}} \overline{b_{n}(z, r)} d z
$$

To be able to apply our result approximately to more realistic model, we include a $\mathrm{z}$ dependence in the bunching parameter, even though in the case of our idealized model $\boldsymbol{b}_{\boldsymbol{n}}(\boldsymbol{r})$ is just the initial bunching parameter independent of $\mathrm{z}$ (in the model there is no energy modulation but only micro-bunching, so the bunching parameter remains constant). The bar represents an average over guided mode $\phi(\boldsymbol{r})$ :

$$
\overline{b_{n}(z)}=\frac{1}{4 \pi \sigma_{x}^{2}} \int b_{n}(z, r) \frac{\phi(r)}{\phi(0)} d^{2} r
$$

The guided mode $\frac{\phi(\boldsymbol{r})}{\phi(\mathbf{0})}=\boldsymbol{J}_{\mathbf{0}}\left(\chi \frac{\boldsymbol{r}}{\boldsymbol{r}_{\mathbf{0}}}\right)$ in our model [7]. In large beam size limit $\chi$ approaches the first root of the Bessel function $\chi \rightarrow \mu_{1} \simeq \mathbf{2 . 4}$.

Now, for more realistic cases, we introduce approximations. In the case of our idealized model, Eq. (3B7) simply gives a product $\mathbf{2} \boldsymbol{L}_{\boldsymbol{G}} \overline{\boldsymbol{b}_{\boldsymbol{n}}}$, then Eq.(3B6) gives an exact solution. But in the more realistic case, the bunching parameter evolves in the radiator due to the existence of the radiator dispersion (the third term of Eq. 3B3), so as an approximation, we replace this product by the integral. For a gaussian transverse profile, we simply model it by a flat top model with the 
same rms beam size $\sigma_{x}$ (i.e., with radius of $2 \sigma_{x}$ ) and same beam current $\boldsymbol{I}_{\mathbf{0}}$. So the integration limit in eq.(3B8) is with radius of $2 \sigma_{x}$.

For the coherent radiation calculation, we ignore energy spread and angular spread, but for the exponential growth section, we take all these into account and use the FEL gain universal scaling function [7] to calculate the gain length $\boldsymbol{L}_{\boldsymbol{G}}$, instead of using the idealized model.

However, we need a further approximation because the energy spread in the radiator section is no longer gaussian due to the energy modulation, even though the gain length calculation given by [7] is very accurate for a gaussian energy spread. As an approximation, we take the energy spread in the radiator to be a quadruture of the initial energy spread and the rms energy modulation at the rms radial beam position:

$$
\sigma_{\gamma 2}=\sqrt{\sigma_{\gamma}{ }^{2}+\left(\frac{\Delta \gamma}{\sqrt{2}}\right)^{2}}
$$

This choice of using $\Delta \gamma$ at the rms radial position $r=\sigma_{x}$, is simply a rough estimate, thus we would not expect the result to be very accurate, but only an estimate.

When the radiator is only two to four gain lengths long, as in our case the first and third stage, there is no exponential growth. We then use eq.(3B6) to calculate the coherent radiation power directly, but when calculate the bunching integral eq.(3B7), the integration limit is the radiator length instead of the two gain lengths mentioned before. Also, since there is no issue of coupling into the guided mode, in the bunching parameter eq.(3B8), the Bessel function $\mathrm{J}_{0}$ is replaced by one.

When the radiator is much longer than a few gain lengths, the estimate eq.(3B5) will have larger error because the gain length estimate (3B9) is not accurate. However, the results still give generally correct behavior for the output power as a function of input power.

\section{B4. The Effect of Finite Emittance in the Dispersion Section is Negligible}

We emphasize here that during the calculation of the bunching parameter $\boldsymbol{b}_{\boldsymbol{n}}$ using Eq.(3B1-4) and coherent radiation power $\boldsymbol{P}_{\boldsymbol{c o h}}$ using Eq.(3B6) we ignore the effect of angular spread, or, in other words, the finite emittance. This effect is only included in the calculation of gain length $\boldsymbol{L}_{\boldsymbol{G}}$ in the exponential growth section. The fact that this approximation gives a good agreement with simulation using the code TDA, as is shown later, is remarkable. This is because the effective energy spread of finite emittace in the dispersion section is negligible. A detailed analysis has been given by Boscolo and Stagno [27]. The main result is rather simple, as explained in the following. 


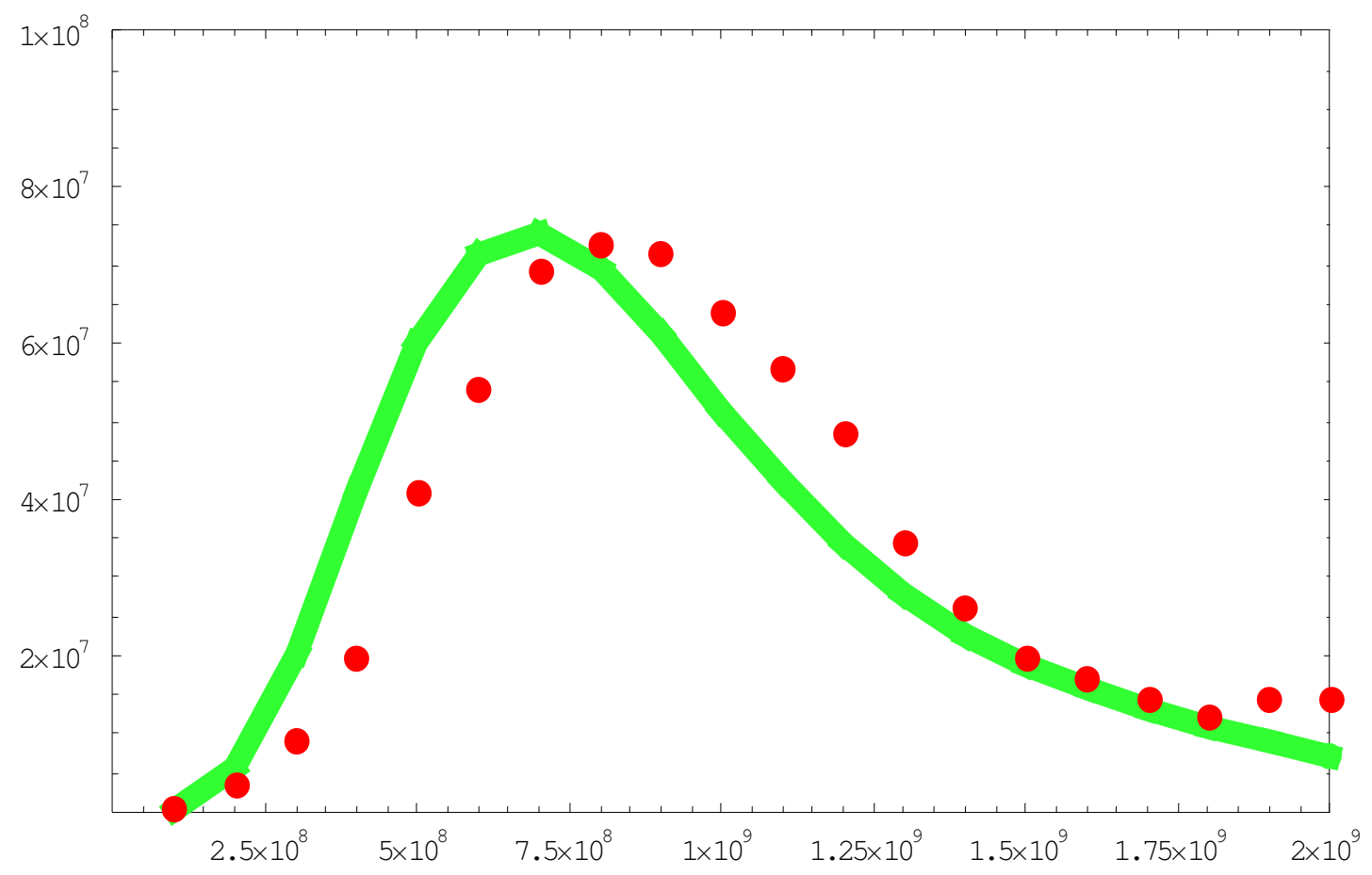

Fig. 4 Output power as function of input power for the third stage. Red dots are simulation using

TDA code. Green curve is the analytical estimate of coherent radiation without exponential growth.

In a chicane magnet used for dispersion section, for a particle with angular deviation $\boldsymbol{x}$, from the reference particle but same energy, the path difference can be calculated with an explicit analytical expression, to second order, it becomes

$$
\Delta s \simeq T_{522} x^{, 2}=\frac{1}{2} s x^{, 2},
$$

where $\boldsymbol{T}_{\mathbf{5 2 2}}$ is the transport matrix element, and s is the path length of the reference electron trajectory. So the net effect of angular spread in a chicane is same as in a drift space of length $\mathrm{s}$, which is only slightly longer than the chicane length itself. However, in the dispersion chicane, the path length difference due to energy spread is much larger than that of the drift space, hence the effective energy spread due to emittance is much smaller than in a drift space.

This result about the insensitivity of coherent radiation process to emittance may have impact on the future works, because the present design of our system may not have taken the full advantage of this.

\section{B5. Saturation Power and Radiator Length}

In order to estimate the radiator or the amplifier length, we need to know the saturation power. The saturation power obtained empirically by fitting simulation results is given by [45], 


$$
P_{s a t}=1.6 \rho\left(\frac{L_{G 1 D}}{L_{G}}\right)^{2} P_{b}
$$

$\mathrm{P}_{\mathrm{b}}=\mathrm{I}_{0} \gamma \mathrm{mc}^{2} / \mathrm{e}$ is the e-beam power. $\mathrm{L}_{\mathrm{G} 1 \mathrm{D}}=\lambda_{w} / \mathbf{4} \pi \sqrt{\mathbf{3}} \rho$ is the 1 - D power e-folding length.

Therefore, if we design the system so that the radiator will extend to the saturation region, then the length of the radiator could be estimated by solving $\mathrm{z}$ from

$$
\boldsymbol{P}=\boldsymbol{C} \quad \boldsymbol{P}_{\text {coh }} \quad \boldsymbol{e}^{\frac{z}{L_{G}}}=\boldsymbol{P}_{\text {sat }}
$$

With such straightforward analytical estimate, we could check the numerical simulation results from TDA. The analytical estimate, though very rough, gives a reasonable agreement with the TDA numerical simulation within a factor of 2 in most cases studied.

\section{B6. Dispersion Magnet}

The dispersion of an idealized chicane magnet (see, for example, reference [18] ) is given by

$$
\frac{d \psi}{d \gamma}=\frac{1}{48} B_{0}^{2} s^{3}\left(\frac{e}{m c}\right)^{2} \frac{2 \pi}{s} \frac{1}{\gamma^{3}}
$$

where $\mathrm{B}_{0}$ is the magnetic field in the chicane, and $\mathrm{s}$ is the total length of the chicane, and $\lambda_{\mathrm{s}}$ is the radiation wavelength of the radiator. This idealized chicane consists of three pieces of magnets with constant field $\mathrm{B}_{0},-\mathrm{B}_{0}, \mathrm{~B}_{0}$, and with length $\mathrm{s} / 4, \mathrm{~s} / 2$, s/4 respectively. For example, $\gamma=5400$ and for the first HGHG stage $\lambda_{\mathrm{s}}=53 \mathrm{~nm}, \mathrm{~d} \psi / \mathrm{d} \gamma=1.0$ (see Table 1), if we choose $\mathrm{B}_{0}=1.5$ Tesla, we find $\mathrm{s}=0.43 \mathrm{~m}$. Similarly we find for the dispersion magnets for second and third stages to have length of $0.25 \mathrm{~m}$ and $0.12 \mathrm{~m}$ respectively. In practicality, we should allow for a slightly longer magnet so we can have larger range of adjustment. As mentioned before, the CSR effect in these magnets are negligible, because the dispersion of these magnets are many order of magnitudes smaller than a compression chicane.

\section{B7. Shifter, Connection Region and Rayleigh Range of the FEL}

So far, we have described the details for the design of one complete stage, i.e. a modulator, a dispersion section, and then a radiator. In the cascading scheme [35-37], we use "fresh bunch technique" [37] to shift the light to a fresh part of the e-beam. The length of the shifter determines the diffraction loss of the harmonic radiation between the exit from one stage to the entrance of the next stage. We need to consider the diffraction effect.

Given the light pulse shift time, say, $\Delta \mathrm{S}$, the corresponding shift distance $\mathrm{L}_{\mathrm{s}}$ reads,

$$
L_{s}=\left(\frac{96 m^{2} c^{2} \gamma^{2} \Delta S}{e^{2} B^{2}}\right)^{\frac{1}{3}}
$$

As usual, we assume the idealized dispersion section as given in section B6 
The diffraction loss is taking into account by calculating the Rayleigh range of the output radiation from the exit of harmonic generation stage. According to Eq. (7) of Ref. [7], the fundamental mode of the FEL is assumed to be a Gaussian within the e-beam, and a Hankel function outside the e-beam. Hence, once we found the fundamental mode, we know the Rayleigh range and the waist position of the FEL. These numbers are used in our simulation, then the coupling of the radiation in the next stage take into account of the diffraction loss.

With the guidance of the analytical estimate described, we use numerical simulation to study the performances of the system as described in the next sections.

\section{Stability and the sensitivity to parameter variation}

\section{Design thought}

We need to check the stability of the performance of this system. For each stage, the fluctuation in any parameter of the e-beam, or the seed laser will lead to the fluctuation of the output power of the harmonic at the end of the radiator. But, this output harmonic is the input seed laser for the next stage. Therefore, the fluctuation in the output power of the harmonic in one stage is just the fluctuation in the input power of the seed laser for the next stage. So, the stability consideration could be simplified, i.e. we need only check whether each stage of HGHG could reduce the fluctuation. To make it more explicit, we need to check whether the fluctuation of the output power of the harmonic in each stage is less than the fluctuation in the input power of the seed laser of the same stage.

In Fig. 4, we plot the relation between the output power and the input power for the third stage. The variation in the output power is about $30 \%$ when the input power changes from $60 \mathrm{MW}$ to $120 \mathrm{MW}$. Thus the fluctuation is reduced. This is an attractive feature of the HGHG scheme. This result is a trade off between better stability and total wiggler length, i.e., if we use lower harmonic number and increase one more stage, the stability will be further improved. Analytical study in the following section shows that such attractive feature holds as long as the harmonic number is not too high. In our scheme, we use harmonic number 5. Now that each stage reduces the fluctuation, we could expect that, the radiation fluctuation caused by the fluctuation in the parameters of the previous stage will be stabilized in the following stage. Therefore, not much fluctuation is expected after the whole three stages. So the stability of the whole system is determined mostly by the last amplifier. Since it is only $12 \mathrm{~m}$ long, compared with the $35 \mathrm{~m}$ long undulator in the SASE scheme (about 20 gain lengths to reach saturation for SASE), the stability of the HGHG scheme is expected to be better than the SASE scheme. The results of the calculation confirmed this.

\section{Analytic consideration}

In order that the system could have good performance, we need consider the stability of the system. So, let us look back at the bunching factor, i.e. Eq. (3B4). The exponential part is monotonous, but the Bessel function has a peak, so we could make use of this property. In the 
bunching factor, once an e-beam is given, $\sigma_{\gamma}$ is fixed, and we are left with two free parameters to adjust. $\Delta \gamma$ is a property from the modulator, while $\mathrm{d} \psi / \mathrm{d} \gamma$ is related to the dispersion section. For simplicity, let $\mathrm{y}=\Delta \gamma(\mathrm{d} \psi / \mathrm{d} \gamma)$, and $\mathrm{n}=5$. As a first thought, we would adjust $\mathrm{y}$, so that $\mathrm{J}_{5}(\mathrm{y})$ reaches maximum, i.e. we would like to put $\mathrm{y} \approx 6.4$. Now, recall that

$$
\Delta \gamma \propto \sqrt{\boldsymbol{P}_{\text {in }}}
$$

according to Eq. (3B1). Let $\mathrm{y}=\sqrt{x}$, clearly $\mathrm{x}$ is a quantity proportional to $P_{i n}$. Now according to Eq.s (3B6), (3B5) and (3B4),

$$
P_{o u t} \propto b_{n}{ }^{2} \propto\left(J_{n}(\sqrt{x})\right)^{2}
$$

This function is plotted in Fig. 5. It is noticed that when $x$ varies from 12 to 24 , the function only changed about $30 \%$. This explained the remark we made in the previous paragraph in regarding to figure 4. Now, suppose, the $\mathrm{d} \psi / \mathrm{d} \gamma$ is tuned, so that, for an input power $\boldsymbol{P}_{\boldsymbol{i n}}^{\text {peak }}$,

$$
\Delta \text { peak } \frac{d \psi}{d}=6.4
$$

where, $\Delta \gamma^{\text {peak }}$ is the energy modulator produced by $\boldsymbol{P}_{\boldsymbol{i n}}^{\text {peak }}$. For the same $\mathrm{d} \psi / \mathrm{d} \gamma$, we can calculate the output power if the input power is $\boldsymbol{P}_{\boldsymbol{i n}}^{\mathbf{1 / 3}}=\boldsymbol{P}_{\boldsymbol{i n}}^{\text {peak }} / 3$. Now, let us calculate the ratio of the two radiation powers. We have

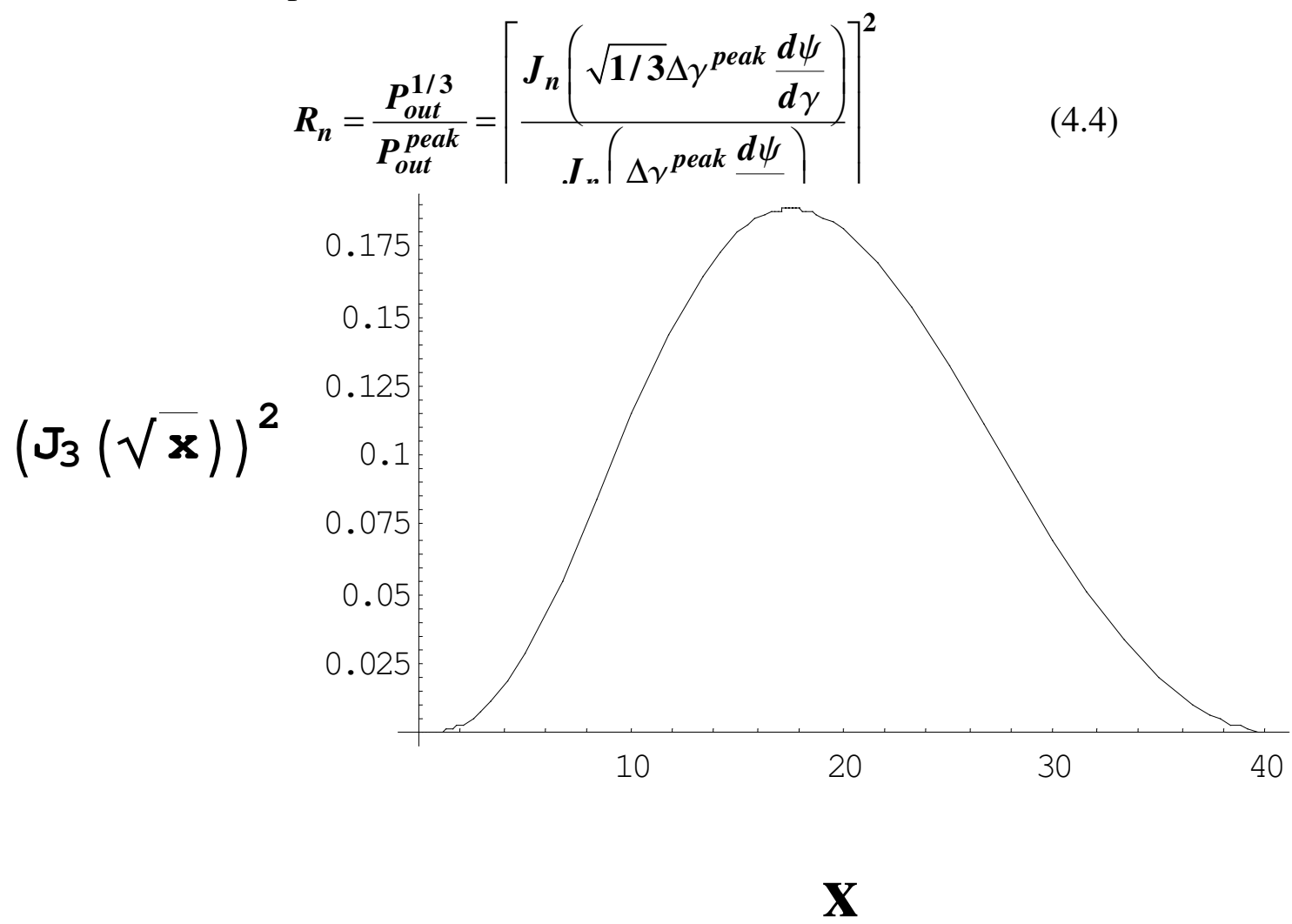

Fig. 5 Use Bessel function to improve stability 
In the case when the dispersion is tuned so that Eq. (4.3) is satisfied for $n=5$, we have $\mathrm{R}_{5} \approx 8 \%$. Thus in this case the system is very sensitive, since we need to use multiple stages, and the fluctuation in the former stage will affect the next stage and so on. So, if we optimize the system according to Eq. (4.3), we would require very small fluctuation in the system. One way to overcome this problem is to make the radiator longer, so that the exponential growth region will be reached. Now, if the input power is smaller, so is the energy modulation, the coherent radiation is smaller, but, the effective energy spread due to the energy modulation is smaller, so the power e-folding length is shorter, i.e. the radiation will grow faster, so somewhere in the radiator, the smaller input power curve will cross the larger input power curve. So, if the power e-folding length of the FEL is small, we would like to make the radiator longer, and even let it go to the saturation region, where the fluctuation is expected to be small. However, due to the effective energy spread from the energy modulation, $\mathrm{L}_{\mathrm{G}}$ is longer than without the energy modulation. Therefore, to make the radiator longer to ensure stability will increase the system length significantly. But, according to Fig. 5, if we overtune the dispersion, so that $\Delta \gamma^{\text {peak }} \mathrm{d} \psi / \mathrm{d} \gamma$ is larger than $6.4, \mathrm{~J}_{5}\left[\Delta \gamma^{\text {peak }} \mathrm{d} \psi / \mathrm{d} \gamma\right]$ will drop, while $\mathrm{J}_{5}\left[\sqrt{\mathbf{1 / 3}} \Delta \gamma^{\text {peak }} \mathrm{d} \psi / \mathrm{d} \gamma\right]$ will become large, so we can make these two numbers equal, i.e. we may have $R_{n}=1$. Or equivalently, we need to solve

$$
J_{n}\left(x^{p e a k}\right)=J_{n}\left(x^{p e a k} / \sqrt{3}\right)
$$

where $\mathrm{x}^{\text {peak }}=\Delta \gamma^{\text {peak }} \mathrm{d} \psi / \mathrm{d} \gamma$, to find the right $\mathrm{d} \psi / \mathrm{d} \gamma$ for a given $\Delta \gamma^{\text {peak }}$. Eq. (4.5) tells us that a factor of 3 difference in the input power will produce the same coherent radiation output power. Now, let us look at the fluctuation in the output power for this $\mathrm{d} \psi / \mathrm{d} \gamma$. Since we overtuned the dispersion, the maximum output power will be produced by some input power smaller than that for $\Delta \gamma^{\text {peak }}$. Let us introduce

$$
n=\frac{P_{\text {out }}^{\text {peak }}}{\left(P_{\text {out }}\right)_{\max }}=\left[\frac{J_{n}\left(\Delta \gamma^{\text {peak }} \frac{d \psi}{d \gamma}\right)}{J_{n}\left(\Delta \gamma \frac{d \psi}{d \gamma}\right)_{\max }}\right]^{2}
$$

In the above formula, "peak" stands for the peak in the input power, while the "max" stands for the "maximum" in the output power in the case of overtuned dispersion. It is found that, $\eta_{5} \approx$ $40 \%, \eta_{4} \approx 50 \%$, and $\eta_{3} \approx 60 \%$. Recall that in such “overtuning" scheme, we would have $\mathrm{R}_{\mathrm{n}}=1$, so the input power varies 3 times from $\boldsymbol{P}_{\boldsymbol{i n}}^{\text {peak }}$ drops to $\boldsymbol{P}_{\boldsymbol{i n}}^{\mathbf{1 / 3}}=\boldsymbol{P}_{\boldsymbol{i n}}^{\text {peak }} / 3$. Therefore, the fluctuation in the output power is smaller than that in the input power for $n \leq 5$.

Since the energy modulation also varies across the transverse section of the e-beam, such "overtuning" mechanism also smoothes the transverse profile of the radiation. For a focused Gaussian seed laser, the on-axis part of the e-beam has the largest energy modulation, while the one at the outside part of the e-beam has smaller energy modulation. A right strength $\mathrm{d} \psi / \mathrm{d} \gamma$ will lead to overbunching on-axis, best bunching at some part of the e-beam, and under-bunching at the outside part. But, the radiation produced by some of the outside part could be the same as that produced by the on-axis part. Therefore the radiation becomes flat, especially after multistage.. 
Such transverse smoothing mechanism leads to a larger Rayleigh region, and it reduces the diffraction loss in the connection region.

The fluctuation in the global or local energy spread, the current, and the emittance all result in the fluctuation in the output power, which is the input power of the next stage. Therefore, by overtuning the dispersion strength, all such fluctuation could be treated in the same way, and the system becocmes less sensitive to parameter variation. Hence this overtuning scheme can improve the stability of the system.

\section{Simulation}

The calculation is carried out by the modified version [18] of TDA code [46], together with analytical estimate presented in the last section. For each stage, the analytical estimates agree with the simulation to about a factor of 2 . In the calculation, we also consider the diffraction effect of the laser when it traverses the "shifter" between stages. As described in section 2, between the 1 st and 2 nd stage, the $2.6 \mathrm{GeV}$ electron bunch needs to be delayed $40 \mathrm{fs}$, for the magnet field $\mathrm{B}=1.5$ Tesla, the "shifter" should be $\mathrm{L}_{\mathrm{s}}=35 \mathrm{~cm}$ long.

As we mentioned before, we choose the betatron function to be $6.8 \mathrm{~m}$ for both horizontal and vertical plane. This based on the assumption that the undulators for the whole system are modular with each section 2 meter long. The spaces between sections are 0.5 meter long, and each 2.5 meter section form one half of a FODO lattice. Simulation showed that the gain lengths for a continuous focusing undulator with beta function of $6.8 \mathrm{~m}$ and for a FODO lattice with same average betatron function (beta maximum is $9.55 \mathrm{~m}$ while beta minimum is $4.09 \mathrm{~m}$ ) of $6.8 \mathrm{~m}$ are nearly the same. For example, for the $2.128 \mathrm{~nm}$ amplifier undulator, the gain length is $1.75 \mathrm{~m}$ for both continuous focusing and FODO focusing.

The output of different stages are given in Figure 3. The total length of the system is 36 meter. Further optimization using the "overtuning" scheme to improve the stability of the system is still under way. A similar calculation has been carried out [44] showing the significant improvement of stability. One byproduct of the improved stability is that because when input power is within a stability window, the output power becomes rather insensitive to the variation of the input power, a gaussian input laser pulse will generate a flat top pulse with pulse length slightly shorter than the input pulse length [44].

\section{Harmonics in the last Amplifier}

The last amplifier is $12 \mathrm{~m}$ long. When saturated, it generates rich harmonics. The $3^{\text {rd }}$ harmonic of the $2.128 \mathrm{~nm}$ radiation at $0.71 \mathrm{~nm}$ is calculated using an upgraded version of TDA, developed by Juhao $\mathrm{Wu}$ [47], to be $2.5 \%$ of the fundamental. Since the $2.1 \mathrm{~nm}$ radiation is $1.7 \mathrm{GW}$ at saturation, the $0.7 \mathrm{~nm}$ radiation is about $44 \mathrm{MW}$. 


\section{Issues on noise degradation in HGHG process}

Saldin et. al. [41] pointed out the shot noise in the undulator may cause a significant degradation of the coherent properties of the HGHG output at very high harmonics. The noise to signal ratio at the input and output satisfies

$$
\left(\frac{P_{n}}{P_{s}}\right)_{o u t}=N^{2}\left(\frac{P_{n}}{P_{s}}\right)_{i n}
$$

where $\mathrm{N}$ is the harmonic number. In their case, in order to generate very small energy modulation, the input laser power is of order of several tens of megawatts. The start-up noise (shot noise) is about 100 watt, and the harmonic number is about 30 to achieve $8 \mathrm{~nm}$, then Eq.(4.7) gives an output noise to signal ratio of about 1\%. Let us consider applying Eq.(4.7) to the scheme proposed here. The scheme employs a fresh bunch technique [37] where after each HGHG stage the (short) laser pulse is shifted to a new (fresh) part of the electron bunch. In this approach, we can generate very large energy modulation at each HGHG stage and generate harmonic radiation at several hundreds megawatts level. The input laser power of the modulator of the first stage is $500 \mathrm{MW}$, while the shot noise is only 30 watt. Therefore, the noise given by Eq.(4.7) using the harmonic number $\mathrm{N}=125$ is $0.1 \%$ of the output signal at the $2.1 \mathrm{~nm}$. The radiator is $6 \mathrm{~m}$ long with SASE output about $600 \mathrm{~W}$, while the output is $400 \mathrm{MW}$ for this stage. Hence the noise given by Eq.(4.7) is also $0.1 \%$ of the output signal at the $2.1 \mathrm{~nm}$. For the second stage, power input is $400 \mathrm{MW}$, but now the harmonic number is lower by a factor of 5 . For other stages, since the harmonic number is further reduced, the contribution to the noise degradation is negligible in our case. Therefore the shot noise in the undulator, even though an important issue, coverts to a noise level at the final output of order of about $0.1 \%$ of the signal.

\section{Challenges}

There are many challenging issues with the development of the soft-x-ray FEL using HGHG scheme. Clearly, the requirement on the electron beam quality is very challenging, in particular the high current and the low emittance. In this section, we emphasize the following issues associated with FEL itself:

1. The synchronization between the electron bunch and the laser seed. The fresh bunch technique overcomes the issue created by the FEL interaction of one HGHG stage by shifting the light pulse to a new and fresh part of the electron bunch, thus allowing strong energy modulation and thus significantly improve the efficiency of the harmonic generation process, and reduces the degradation effect of the shot noise. However, this requires that the jitter between the laser and the electron bunch to be much smaller than the bunch length. For a bunch length of $500 \mathrm{fs}$, and input laser pulse length of $20 \mathrm{fs}$, we assume the shifters shift the light pulse $40 \mathrm{fs}$ between stages. Thus the total used part of the electron bunch after the electron bunch passes through the whole system is about 200 fs. Hence the jitter between the laser and the electron bunch should be less than $100 \mathrm{fs}$. The current system used for our UVFEL has a time jitter about $0.5 \mathrm{ps}$. A new scheme has 
been developed to accurately synchronize the electron bunch and the seed laser pulse. The basic idea is to use the UVFEL output generated in the $800 \mathrm{~nm}-266 \mathrm{~nm}$ HGHG process as second photo-cathode drive laser pulse [42]. Since this output pulse is synchronized with the electron bunch and the RF phase, the electron bunch generated by this pulse is also synchronized to the seed split from the same output pulse. This method is expected to reduce the jitter from 0.5 ps to way bellow 50 fs. The DUVFEL program provides an opportunity to carry out such a research and development project.

2. Possible enhanced start-up noise due to bunch compression process. In the bunch compression process, the structures in the longitudinal phase space of the electron bunch can be amplified and creates enhanced micro-bunching with components in the seeding wavelength range of $266 \mathrm{~nm}$. This micro-bunching produces coherent radiation in the beginning section of the undulator which is then amplified exponentially, and evidenced itself as an enhanced start-up noise. Again, the current DUVFEL provides an opportunity for this investigation. Recent success of the $800 \mathrm{~nm}$ to $266 \mathrm{~nm}$ HGHG experiment showed that the HGHG output is nearly Fourier-transform limited. At the end of the 10 meter radiator NISUS the spectral brightness of the HGHG output is $2 \times 10^{5}$ times larger than the background SASE signal. Since the system is saturated at 5 meter in the NISUS where the SASE signal is 500 smaller, if we terminate the NISUS at 5 meters, the signal noise ratio would be $10^{8}$. Substituted this ratio into Eq.(4.7), we see that the noise to signal ratio at soft $\mathrm{x}$-ray region is very small.

In addition to this, one possible method to further reduce the noise is to remove energy chirping, and then send the electron bunch through a chicane to wash out the microstructure in the electron bunch. This may also help to reduce the damaging effect of the coherent synchrotron radiation in the compression process

3. Undulator trajectory error tolerance. The most stringent requirement for trajectory in the undulator is determined by the 12 meter $2.1 \mathrm{~nm}$ amplifier $\left(\mathrm{L}_{\mathrm{w}}=12 \mathrm{~m}, \lambda_{\mathrm{s}}=2.1 \mathrm{~nm}\right)$. We assume that, as described before, the undulator system consists 2 meter long modular sections separated by 0.5 meter spaces, and there are position monitors in the middle of modular sections so that the trajectory can be corrected every $\mathrm{L}_{\mathrm{s}}=1.25$ meter. The gain length is $\mathrm{L}_{\mathrm{G}}=1.75 \mathrm{~m}$. When the spacing between the correction station $\mathrm{L}_{\mathrm{s}}$ is smaller than the gain length $\mathrm{L}_{\mathrm{G}}$ as is our case, the trajectory tolerance is derived from a formula given in the reference [8]:

$$
x_{t o l}=\left(\frac{L_{s}}{L_{G}}\right)^{\frac{3}{4}} \times 0.266 \sqrt{\lambda_{s} L_{G}}\left(\frac{L_{G}}{L_{w}}\right)^{\frac{1}{4}}
$$

This formula has been compared with simulation for many different occasions, the agreement is within about $40 \%$. When we substitute the parameters into this equation, we find the tolerance is $8 \mu \mathrm{m}$. If the trajectory is deviating from the axis by more than this, the output power is dropped by half. For a single pass FEL with exponential growth, this is acceptable loss which can be compensated by appropriate increase of the length of the undulator. But this tolerance still give an rough estimate of the allowable tolerance on the trajectory. The present diagnostic system based on HeNe laser alignment in the DUVFEL has achieved reproducibility and resolution of $20 \mu \mathrm{m}$. We need to further improve the 
quality of the HeNe profile images in the undulator to obtain a better resolution. Because the present resolution is determined mostly by the defects of the YAG crystal of the beam position monitors, and the mechanical reproducibility of the position of the BPM, this improvement can be achieved. Another important direction for trajectory correction is the beam based alignment procedure being developed now for the DUVFEL[48]. Because ebeam size is much smaller than the HeNe beam size, the resolution will clearly be better. The analysis also provides information about the trajectory between monitors. Therefore the DUVFEL program provides another opportunity for us to meet the challenges.

\section{Conclusion}

In conclusion, the cascading HGHG scheme is an attractive scheme to generate coherent soft-xray. It will have the advantages mentioned in the beginning of the chapter. Among them, the most attractive feature is that, the HGHG FEL will provides an intense output as short as 20fs, another advantage is its stability and its longitudinal coherent output. There are many challenging issues associated with this scheme, the DUVFEL program provides an excellent opportunity to study these issues.

\section{References}

1. Ya.S. Derbenev, A.M. Kondratenko, and E.L. Saldin, Nucl. Instrum. Methods Phys. Res.A, 193 , 415(1982).

2. R. Bonifacio, C. Pellegrini, and L.M. Narducci, Opt.Commun. 50, 373(1984).

3. J.M. Wang, and L.H. Yu, Nucl. Instrum. Methods Phys. Res. A250, 484(1986).

4. K.J. Kim, Nucl. Instrum. Methods Phys. Res. A250, 396(1986).

5. K.J. Kim, Phys. Rev. Lett.57, 1871(1986).

6. S. Krinsky, and L.H. Yu, Phys. Rev. A35, 3406(1987).

7. L.H. Yu, S. Krinsky, and R.L. Gluckstern, Phys. Rev. Lett. 64 , 3011(1990).

8. L.H. Yu, S. Krinsky, R.L. Gluckstern, and J.B.J. van Zeijts, Phys. Rev. A45, 1163(1992).

9. R. Bonifacio, L. De Salvo, P. Pierini, N. Piovella, and C. Pellegrini, Phys. Rev. Lett. 73, 70(1994).

10. Li Hua Yu, Phys. Rev. E 58, 4991(1998).

11. S. Krinsky, Phys. Rev. E 59, 1171(1999).

12. B.W.J. McNeil, G.R.M. Robb, D.A. Jaroszynski, Opt. Comm.165 , 65(1999)

13. SASE gain of $10^{5}$ at $12 \mu \mathrm{m}$ was reported in M.J. Hogan, et al. Phys. Rev. Lett. 82, 4867(1998).

14. SASE gain at $530 \mathrm{~nm}$ was recently observed at the LEUTL facility at APS/ANL in S.V. Milton, et al., Phys. Rev. Lett. 85,988(2000), and at $109 \mathrm{n} \mathrm{m}$ at TTF/DESY in J. Andruszkow, et al., Phys. Rev. Lett. 85, 3825(2000).

15. "Linac Coherent Light Source (LCLS) Design Study Report," The LCLS Design Study Group, Standford Linear Accelerator Center (SLAC) Report No. SLAC-R-521(1998).

16. "TESLA Technical Design Report", The TESLA-N Study Group, DESY-TESLA-200123, ECFA-2001-209, March 2001

17. I. Ben-Zvi, L.F. Di Mauro, S. Krinsky, M.G. White, and L.H. Yu, Nucl. Instrum. Methods Phys. Res. A304, 151(1991).

18. L.H. Yu, Phys. Rev. A44, 5178(1991). 
19. I. Ben-Zvi, A. Friedman, C.M. Hung, G. Ingold, S. Krinsky, and L.H. Yu, Nucl. Instrum. Methods Phys. Res. A318, 208(1992).

20. L.-H. Yu, M. Babzien, I. Ben-Zvi, L.F. DiMauro, A. Doyuran, W. Graves, E. Johnson, S. Krinsky, R. Malone, I. Pogorelsky, J. Skaritka, G. Rakowsky, L. Solomon, X.J. Wang, M. Woodle, V. Yakimenko, S.G. Biedron, J.N. Galayda, E. Gluskin, J. Jagger, V. Sajaev, I. Vasserman, Nucl. Instrum. Methods Phys. Res. A, 445, 301(2000).

21. L.-H. Yu, M. Babzien, I. Ben-Zvi, L.F. DiMauro, A. Doyuran, W. Graves, E. Johnson, S. Krinsky, R. Malone, I. Pogorelsky, J. Skaritka, G. Rakowsky, L. Solomon, X.J. Wang, M. Woodle, V.Yakimenko, S.G. Biedron, J.N. Galayda, E. Gluskin, J. Jagger, V. Sajaev, I. Vasserman, Science, 289 (5481), 932(2000).

22. N. Vinokurov, and Skrinsky, preprint INP 78-88, Novosibirsk (1978).

23. P. Czonka, Part. Accel. 8 , 225(1978).

24. V. Stagno, G. Brautti, T. Clauser, and I. Boscolo, Nuovo Cim. 56B , 219 (1980)

25. W.B. Colson, IEEE J. Quant. Electron. QE-17, 1417(1981).

26. R. Coisson, and F. DeMartini, in Physics of Quantum Electronics Vol. 9, Eds. S.F. Jacobs, M. Sargent III, and M.O. Scully, P. 939, Addison-Wesley, Reading, MA, (1982).

27. I. Boscolo, and V. Stagno, Nucl. Instrum. Methods Phys. Res. A198, 483 (1982).

28. W.B. Colson, and P. Elleaume, Appl. Phys. B 29, 101(1982).

29. I. Schnitzer, and A. Gover, Nucl. Inctrum. Methods Phys. Res. A237, 124 (1985).

30. R. Bonifacio, L. De Salvo Souza, P. Pierini, and E.T. Scharlemann, Nucl. Instrum. Methods Phys. Res. A296, 787 (1990).

31. R. Prazeres, P. Guyot-Sionnest, J.M. Ortega, D. Jaroszynski, M. Billardon, M.E. Couprie, M. Velghe, and Y. Petroff, Nucl. Instrum. Methods Phys. Res. A304, 72 (1991).

32. .R. Bonifacio, R. Corsini, and P. Pierini, Phys. Rev. A45, 4091 (1992).

33. D.A. Jaroszynski, R. Prazers, F. Glotin, O. Marcouille, J.M. Ortega, Nucl. Instrum. Methods Phys. Res. A375, 456 (1996).

34. E.L. Saldin, E.A. Schneidmiller, and M.V. Yurkov, Nucl. Instrum. Methods Phys. Res. A 381, 545(1996)

35. L.H. Yu, Proceedings of the IFCA Advanced Beam Dynamics Workshop on Future Light Sources, C.E. Eyberger, Ed., Argonne National Laboratory, Argonne, IL(1999) (URL: http: //www.aps.anl.gov /conferences /FLSworkshop /proceedings /papers /wg1-01.pdf).).

36. Juhao $\mathrm{Wu}$, and Li Hua Yu, Proc. 22nd International FEL Conference, Duke University, Durham, NC, August 13-18, (2000), BNL-67732, Nucl. Instrum. Methods Phys. Res. A, in press (2001).

37. L.H. Yu, and Ilan Ben-Zvi, Nucl. Instrum. Methods Phys. Res. A393, 96(1997).

38. G. Dattoli, and P.L. Ottaviani, J. Appl. Phys., 86, 5331(1999).

39. Juhao $\mathrm{Wu}$, and $\mathrm{Li}$ Hua $\mathrm{Yu}$, (in preparation).

40. I. Ben-Zvi, K.M. Yang, and L.H. Yu, Nucl. Instrum. Methods Phys. Res. A318, 726(1992).

41. E.L. Saldin, E.A. Schneidmiller, and M.V. Yurkov, Opt. Commun. 202, 169 (2002

42. L.H.Yu, "Accurate synchronization between short laser pulse and electron bunch by using HGHG output at $266 \mathrm{~nm}$ as photo-cathode laser for RF-gun”, BNL-71194-2003-IR (2003)

43. L. H. Yu, L.F. DiMauro, A. Doyuran, W. Graves, E. Johnson, S. Krinsky, S. Mikhailov, G. Rakowsky, J. Skaritka, T. Shaftan, B.Sheehy, J.H.Wu, ”The DUV-FEL Development Program", Proc. PAC 2001, Chicago p2830 (2001).

44. Juhao Wu, L. H. Yu, Nucl. Instru. Meth. A475, 104 (2001) 
45. K.J. Kim, and M. Xie, Nucl. Instrum. Methods Phys. Res. A 331, 359(1993).

46. T.M. Tran, and J.S. Wurtele, Comput. Phys. Commun. 54 , 263(1989).

47. Juhao $\mathrm{Wu}$, private communication

48. H. Loos et al, "Beam-Based Trajectory Alignment in the NISUS Wiggler", Proc. EPAC 2002, Paris, June 3-7, 2002, p. 837. 